\title{
IMPACT FORCE RECONSTRUCTION USING WAVELET DECONVOLUTION TECHNIQUE
}

\author{
Hai Tran ${ }^{1}$ and Hirotsugu Inoue ${ }^{2}$ \\ ${ }^{1}$ Department of Mechanical and Control Engineering, School of Science and Engineering, Tokyo Institute of \\ Technology, Japan. e-mail: tran.h.ab@m.titech.ac.jp \\ ${ }^{2}$ Department of Mechanical Engineering, School of Engineering, Tokyo Institute of Technology, Japan. \\ e-mail: inoue.h.ab@m.titech.ac.jp
}

Received Date: August 28, 2017

\begin{abstract}
Reconstruction or deconvolution of impact force history from corresponding impact responses such as strain, acceleration, and displacement has been considered as a useful indirect method for measuring the impact force. However, due to the ill-posed nature of deconvolution problem, impact force is often inaccurately and unstably reconstructed. This paper introduces and applies the deconvolution technique using wavelets as a robust method for reconstructing impact force with the advantageous properties of wavelets. First, an analytical process of impact force reconstruction by using the wavelet technique in terms of scaling and translating the Haar wavelet is formulated. The unknown impact force is represented by the expanded coefficients at different scales and shifts of Haar wavelet which is compactly supported in the time domain (finite in time). Then, based on the governing equation of impact force deconvolution, the reconstruction process of these expanded coefficients is formulated. Second, a structural model is built by finite element method to obtain impulse response function numerically. After that, the wavelet technique is applied to reconstruct the impact forces acting on the structure to verify its effectiveness. The comparisons between reconstructed forces and finite element analysis results demonstrate the success of the present technique in accurately reconstructing the numerical impact forces acting on the thin-walled column. These achievements show remarkable ability of the wavelet technique for reconstructing accurately any input forces.
\end{abstract}

Keywords: Deconvolution, Haar wavelet, Impact force, Inverse problem, Wavelet transform

\section{Introduction}

There exist countless situations in which direct measurements of impact force are challenging or impossible. For instance, measurement of impact force acting on the specimen in Charpy impact test is required in order to understand the specimen behavior during impact event in detail. In addition, the measurement of force acting on energy absorbing structures under crash test is essential for evaluating the performance. In consideration of this matter, indirect determination of impact force from corresponding response such as strain, displacement, etc. has been paid attention and known as inverse analysis or deconvolution for impact force reconstruction. During the last three decades, there have been many investigations on impact force reconstruction, which are reviewed and summarized thoroughly by Inoue et al. [1], Sanchez and Benaroya [2].

It is well known that a simple reconstruction of impact force is often erroneous and unacceptable because of error amplification due to the ill-posed or ill-conditioned nature. To deal with this difficulty, several regularization techniques such as truncated singular value decomposition or Tikhonov regularization have been applied extensively. In addition, reconstruction from two or more responses using least squares method has been verified 
effective [3]. Recently, Khoo et al. [4] used the pseudo-inverse method to identify impact force acting on a lightweight structure associated with the evaluation of good location for reconstruction process. Gunawan [5] applied the Wiener filter for impact force reconstruction. Qiao et al. [6] studied impact identification using cubic B-spline based on multi-resolution analysis.

The use of wavelets in impact force reconstruction has been also examined in developing a robust method. Doyle [7, 8] applied wavelets to identify impact forces acting on a beam and a plate. Unfortunately, in these papers, the wavelet deconvolution was used only by translating a basic triangular wavelet. However, according to the fundamentals of wavelet theory [9], it is advantageous to employ not only translation but also scaling of the basic wavelet. Inoue et al. [10] showed the effectiveness of the wavelet technique in the inverse analysis of impact force using Gabor wavelet. Lifschitz et al. [11] also studied the use of wavelets for input force estimation, but it was specialized for pulsed plasma thruster. In this reference, the wavelet analysis of the reconstruction process is not really detailed and there is no specific explanation for choosing the basic wavelet "daub4" from Daubechies family. Li et al. [12] identified a load based on wavelet multi-resolution analysis, but they exploited this technique for estimating not impact force but periodic dynamic force. Reconstruction of impact force with wavelets is still a young research, although wavelets have been utilized in many fields of science and engineering. Therefore, this investigation proposes a more comprehensive analysis of wavelet deconvolution technique for reconstructing impact force.

\section{Conventional Deconvolution Technique}

During the impact process, if the impact response $e(t)$ at a specific location of a structure is considered to be linearly dependent on the impact force $f(t)$, this correlation can be expressed as a linear convolution integral

$$
e(t)=\int_{0}^{t} h(t-\tau) f(\tau) d \tau,
$$

where $h(t)$ is the impulse response function, which characterizes impact behavior of the linear system. If $h(t)$ is known in advance and if $e(t)$ can be measured, the applied force $f(t)$ is estimated by solving the deconvolution problem from Equation (1). By normalizing the time $t$ with a constant time $t_{0}$ as $x=t / t_{0}$ and discretizing the normalized time $x$ with a constant period $\Delta x$ (namely, $\Delta x=\Delta t / t_{0}$ ) as $k=x / \Delta x$, the normalized and discrete version of Equation (1) can be expressed as

$$
\{e\}=[h]\{f\},
$$

in which $0 \leq k \leq K-1 ; K$ is the number of discrete points; $\{e\}$ and $\{f\}$ are column vectors as

$$
\{e\}=\{e(0) \quad e(1) \quad e(2) \quad \cdots \quad e(K-1)\}^{T}
$$

and

$$
\{f\}=\{f(0) \quad f(1) \quad f(2) \quad \cdots \quad f(K-1)\}^{T},
$$

respectively; $[h]$ is the impulse response matrix in the following form 


$$
[h]=\left[\begin{array}{cccccc}
h(0) & 0 & 0 & \cdots & 0 & 0 \\
h(1) & h(0) & 0 & \cdots & 0 & 0 \\
h(2) & h(1) & h(0) & \cdots & 0 & 0 \\
\vdots & \vdots & \vdots & \ddots & \vdots & \vdots \\
h(K-2) & h(K-3) & h(K-4) & \cdots & h(0) & 0 \\
h(K-1) & h(K-2) & h(K-3) & \cdots & h(1) & h(0)
\end{array}\right] .
$$

Since the matrix $[h]$ is commonly known to be ill-conditioned, a small error within the response may be amplified into a large disturbance in impact force history. Due to this matter, the conventional deconvolution often leads to unsatisfactory reconstruction of impact force. As well known, the truncated singular value decomposition or the Tikhonov regularization is often employed for stable reconstruction, but it is preferable to develop a more robust technique before applying those regularizations.

\section{Formulation of Wavelet Deconvolution Technique}

There are many wavelet functions which can be applied to represent any signals. However, Haar wavelet has been known as the simplest and useful in many fields of science and engineering. In addition, it has a compact support in the time domain, which is appropriate to represent the impact force normally with a finite duration. Thus, in this work, the Haar wavelet is chosen and employed as the basic wavelet. It is described by

$$
\psi(x)=\left\{\begin{array}{rc}
1, & 0 \leq x<\frac{1}{2} \\
-1, & \frac{1}{2} \leq x<1 \\
0, & \text { otherwise }
\end{array}\right.
$$

as a function of the normalized time $x$ and illustrated as shown in Figure 1 .

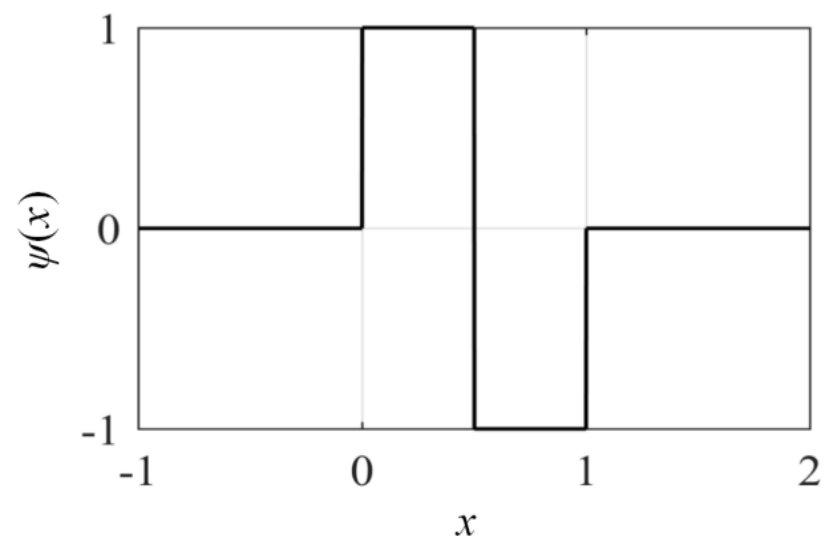

Figure 1. The Haar wavelet function

As confirmed by the wavelet theory [9], wavelets are yielded by shifting and scaling the basic wavelet function $\psi(x)$ such as

$$
\psi_{m, n}(x)=a_{0}^{-\frac{m}{2}} \psi\left(\frac{x-n b_{0} a_{0}^{m}}{a_{0}^{m}}\right),
$$


where $a_{0}^{-m}$ and $n b_{0} a_{0}^{m}$ are the scale and translation parameters, respectively; $a_{0}^{-\frac{m}{2}}$ is a normalization constant; $m$ and $n$ are integers. By using wavelets, any impact force $f(x)$ can be represented by the following summation

$$
f(x)=\sum_{m} \sum_{n} \tilde{f}_{m, n} \psi_{m, n}(x) .
$$

Substituting Equation (8) into Equation (1) with the normalized time $x$, it yields

$$
e(x)=\sum_{m} \sum_{n} \tilde{f}_{m, n} \phi_{m, n}(x),
$$

in which $\phi_{m, n}(x)$ is considered as the response to each wavelet force $\psi_{m, n}(x)$, and it is defined by

$$
\phi_{m, n}(x)=\int_{0}^{x} h(x-\xi) \psi_{m, n}(\xi) d \xi .
$$

In actual computation, since the response data have a finite duration, the range of scale level $(m)$ and the number of shifts $(n)$ of the wavelet in the above equations should be truncated. Assuming that the data consist of $K$ samples, the scales and translations of the Haar wavelet are respectively limited by

$$
1 \leq m \leq \log _{a_{0}} K=M
$$

and

$$
0 \leq n \leq \frac{K}{b_{0} a_{0}^{m}}-1=N_{m}
$$

Note that in Equations (11) and (12), the largest level of scale and the last shift of the wavelet are denoted as $M$ and $N_{m}$, respectively. Using dyadic grid with $a_{0}=2$ and choosing $b_{0}=1$, the discrete value $K$ should be chosen as $K=2^{M}$ so that $M$ and $N_{m}$ become integers. Then, Equation (8) can be presented in vector and matrix form as

$$
\{f\}=[\Psi]\{\tilde{F}\},
$$

and Equation (9) can be discretized as

$$
\{e\}=[\Phi]\{\tilde{F}\} \text {. }
$$

In the above equations, $\{\tilde{F}\}$ is a column vector whose elements are $\tilde{f}_{m, n}$ with row indices $m$ and $n$

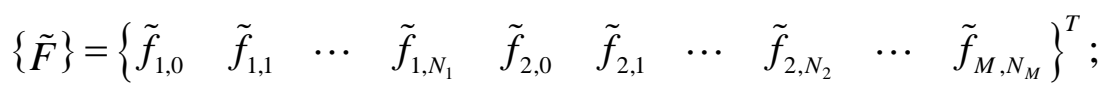

$[\Psi]$ and $[\Phi]$ are matrices composed of $\psi_{m, n}(k)$ and $\phi_{m, n}(k)$, respectively, in which $k$ is row index; $m$ and $n$ are column indices as follows

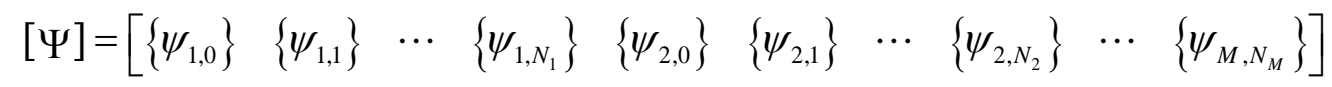

and 


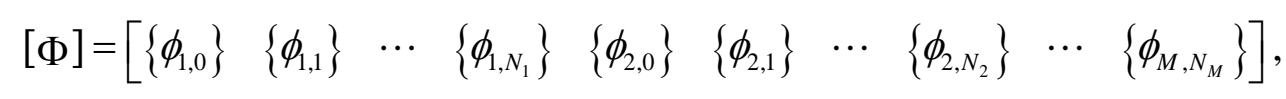

where

$$
\left\{\psi_{m, n}\right\}=\left\{\psi_{m, n}(0) \quad \psi_{m, n}(1) \quad \psi_{m, n}(2) \quad \cdots \quad \psi_{m, n}(K-1)\right\}^{T}
$$

and

$$
\left\{\phi_{m, n}\right\}=\left\{\phi_{m, n}(0) \quad \phi_{m, n}(1) \quad \phi_{m, n}(2) \quad \cdots \quad \phi_{m, n}(K-1)\right\}^{T} .
$$

Based on Equations (13) and (14), the impact force history can be reconstructed from the response by

$$
\{f\}=[\Psi][\Phi]^{+}\{e\},
$$

where $[\Phi]^{+}$is the Moore-Penrose generalized inverse of $[\Phi]$.

Additionally, in the case of incorporating several responses at different locations, Equation (20) can be developed and written as

$$
\{f\}=[\Psi]\left[\begin{array}{c}
{\left[\Phi_{1}\right]} \\
{\left[\Phi_{2}\right]} \\
\vdots \\
{\left[\Phi_{L}\right]}
\end{array}\right]^{+}\left\{\begin{array}{c}
\left\{e_{1}\right\} \\
\left\{e_{2}\right\} \\
\vdots \\
\left\{e_{L}\right\}
\end{array}\right\},
$$

of which $l(1 \leq l \leq L)$ denotes $l$ th response location.

As above expression, [ $\Phi]$ can be obtained as a product of the impulse response matrix $[h]$ and the matrix $[\Psi]$ as $[\Phi]=[h][\Psi]$, in which $[\Psi]$ consists of vectors representing wavelets as shown in Equation (16). In the same manner as the introduction given by Qiao et al. [6] and Gunawan et al. [13], due to strong diagonal entities in the matrix $[\Psi]$, it could make the improvement of ill-posed nature of impact force reconstruction problem in spite of the ill-conditioned of the matrix $[h]$. Thus, this possibility of $[\Psi]$ in relaxing the ill-posedness of deconvolution method can be regarded as an advantage of the present technique in order to provide a more robust reconstruction technique using wavelets. It should be added here that the ability of the present approach in reconstructing impact force at different scales is also an interesting aspect which can be considered to enhance the reconstructed result.

In addition, it is needed to note that $[\Phi]$ in the above equations can be constructed only when the impulse response function has been already known. In this sense, the impact force and the impact response must be identified in advance to obtain the impulse response function. Particularly, in this work, this impulse response function is also determined by using the wavelet deconvolution when both the impact force and the impact response are given by numerical simulation. In the same manner as the reconstruction of impact force described above, the estimation of impulse response function can be briefly provided by

$$
\{h\}=[\Psi][\Omega]^{+}\{e\},
$$

where $[\Omega]$ is set up in a similar manner as $[\Phi]$, and its elements $\omega_{m, n}(x)$ are given by 


$$
\omega_{m, n}(x)=\int_{0}^{x} f(x-\xi) \psi_{m, n}(\xi) d \xi .
$$

\section{Verification of Wavelet Deconvolution Technique}

\section{Numerical Model}

A drop impact testing of square tube studied in references [14, 15] is considered as an application example in this study. Figure 2(a) depicts the finite element model, which is composed of three parts: the impactor, the square tube, and the load cell. The material properties of these parts are listed in Table 1. The square tube is placed upright on the top surface of the load cell and then subjected to impact at its top end vertically by the impactor with an initial velocity $V$. The bottom of the load cell is fixed in all directions. The numerical simulation was conducted by LS-DYNA.

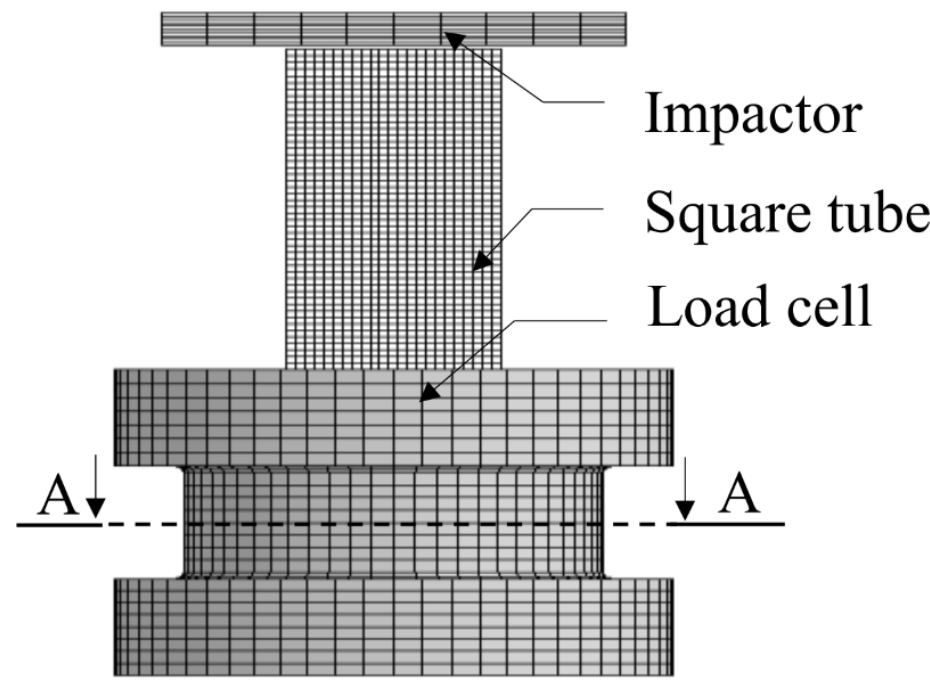

(a) FE impact model

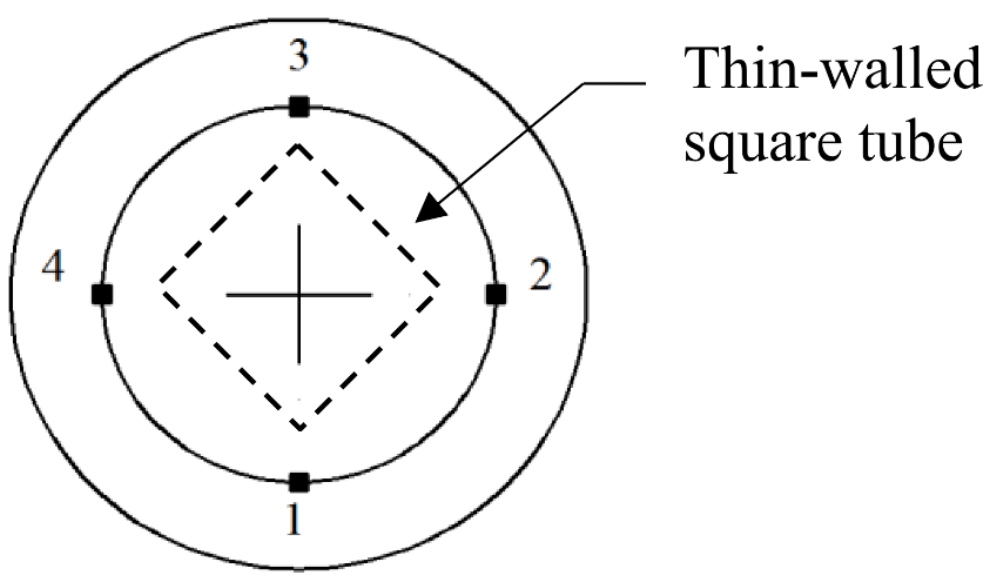

(b) Four locations to measure strain responses at section A-A on the load cell part

Figure 2. Drop impact model of the thin-walled square tube and the measured response locations on the load cell body 
Table 1. Material Parameters of Simulation Model

\begin{tabular}{cccc}
\hline Parameters & Square Tube [14] & Load Cell [15] & Impactor \\
\hline Young's modulus [GPa] & 188 & 200 & 200 \\
Mass density [kg/m ${ }^{3}$ ] & 7330 & 7770 & 7770 \\
Poisson's ratio & 0.3 & 0.3 & 0.3 \\
Yield stress [MPa] & 186.95 & - & - \\
Ultimate tensile strength [MPa] & 289.56 & - & - \\
\hline
\end{tabular}

For a tube with length: $100 \mathrm{~mm}$, thickness: $1.0 \mathrm{~mm}$ and cross-section: $46 \mathrm{~mm}$ square and for the impact velocity $V=6 \mathrm{~m} / \mathrm{s}$, the total impact force acting between the square tube and the load cell was obtained as shown in Figure 3(a), while the corresponding strain response in the longitudinal direction at Location 1 in Figure 2(b) was obtained as shown in Figure 3(b). Although the load cell is designed well enough and hence the strain response is similar (in opposite sign) to the impact force, the strain response is more oscillatory than the impact force.

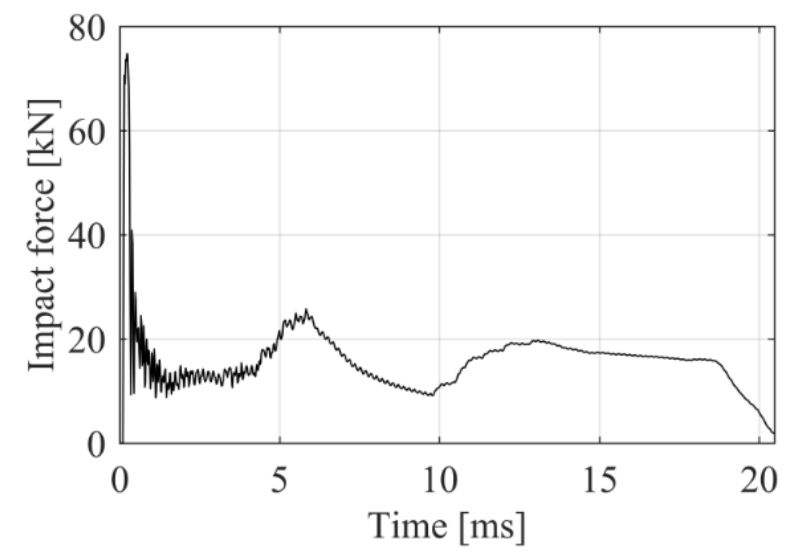

(a) The total impact force acting between the tube and the load cell

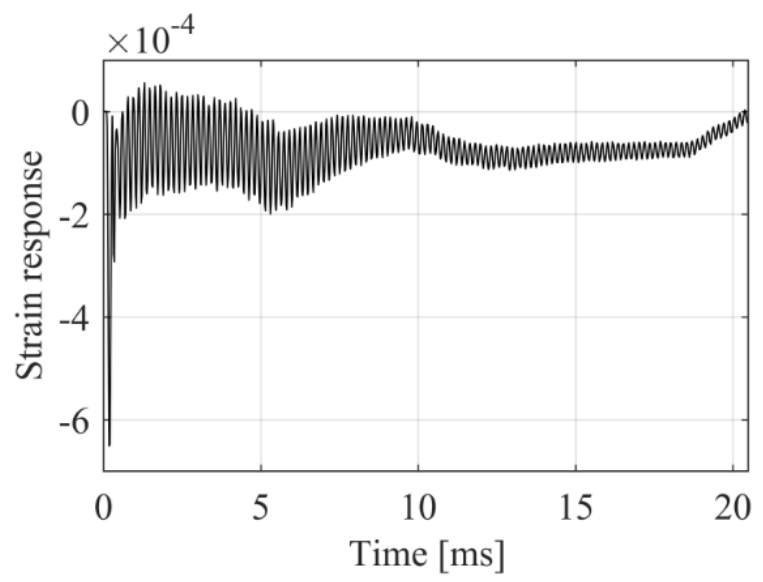

(b) Strain response at Location 1 in the longitudinal direction of the load cell

Figure 3. The results of numerical simulation for square tube: $100 \mathrm{~mm}$ long $\times 1.0 \mathrm{~mm}$ thick $\times 46 \mathrm{~mm}$ square cross-section, and $V=6 \mathrm{~m} / \mathrm{s}$ 


\section{Reconstruction of Impact Force}

In the numerical simulation of impact force reconstruction, the impact time was $t=20.48$ ms; the sampling period and the normalized time were respectively set to $\Delta t=20 \mu$ s and $t_{0}=\Delta t / 2$; the total discretize points $K=1024$. The number of shifts $(n)$ of the Haar wavelet depends on its corresponding scale levels $(\mathrm{m})$ and those are calculated by Equations (11) and (12). First, the impulse response function was determined numerically from the reference impact force and the reference strain response shown in Figures 3(a) and 3(b), respectively, by deploying the wavelet deconvolution technique. Then the impact force was reconstructed from the strain response shown in Figure 3(b). The result shown in Figure 4 is similar to the original shown in Figure 3(a) in the first half but it is quite noisy in the latter half, which must be due to the ill-conditioned nature of the reconstruction problem.

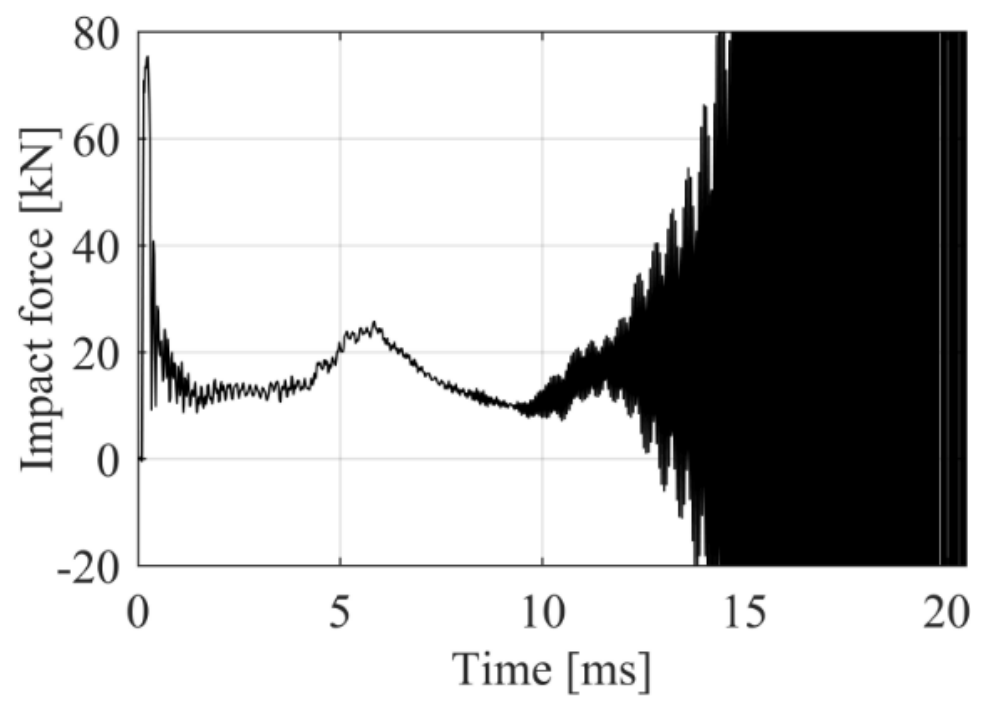

(a) The whole impact time

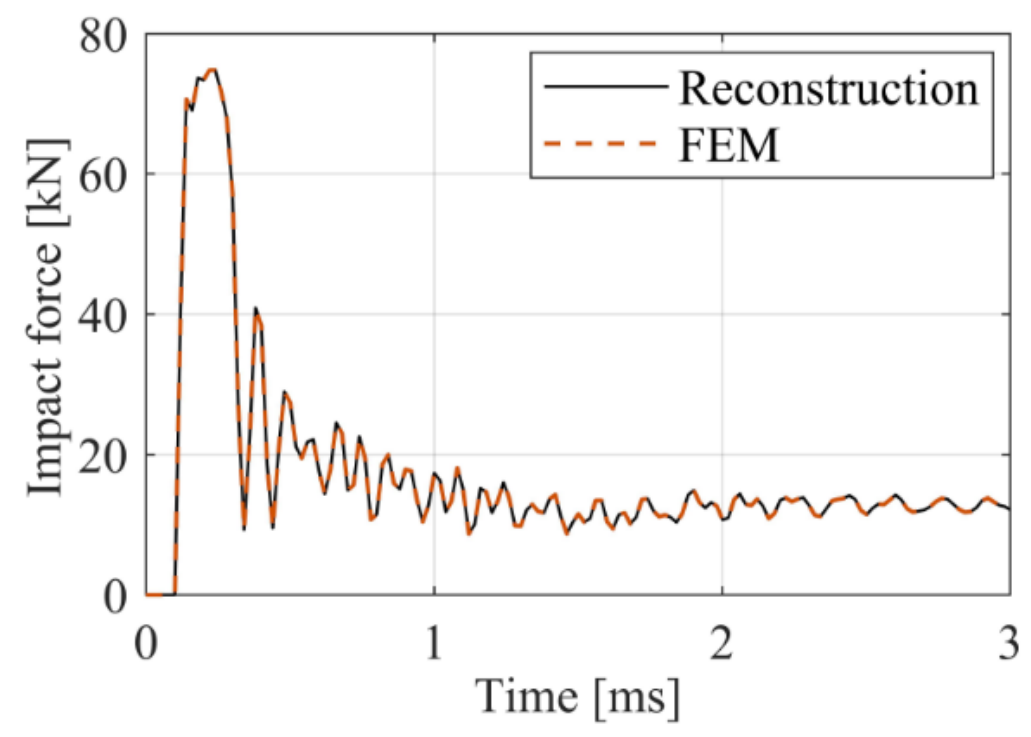

(b) Magnification for the first $3 \mathrm{~ms}$

Figure 4. Reconstructed impact force for square tube: $100 \mathrm{~mm}$ long $\times 1.0 \mathrm{~mm}$ thick $\times 46$ $\mathrm{mm}$ square cross-section, and $V=6 \mathrm{~m} / \mathrm{s}$ using the strain response at Location 1 
Next, the same impact force was reconstructed from all four strain responses obtained at Locations 1 to 4 in Figure 2(b) according to Equation (21). The result presented in Figure 5 coincides very well with the original for all time range computed. It can be judged that the use of multiple responses is effective to reduce the noise expansion in impact force reconstruction.

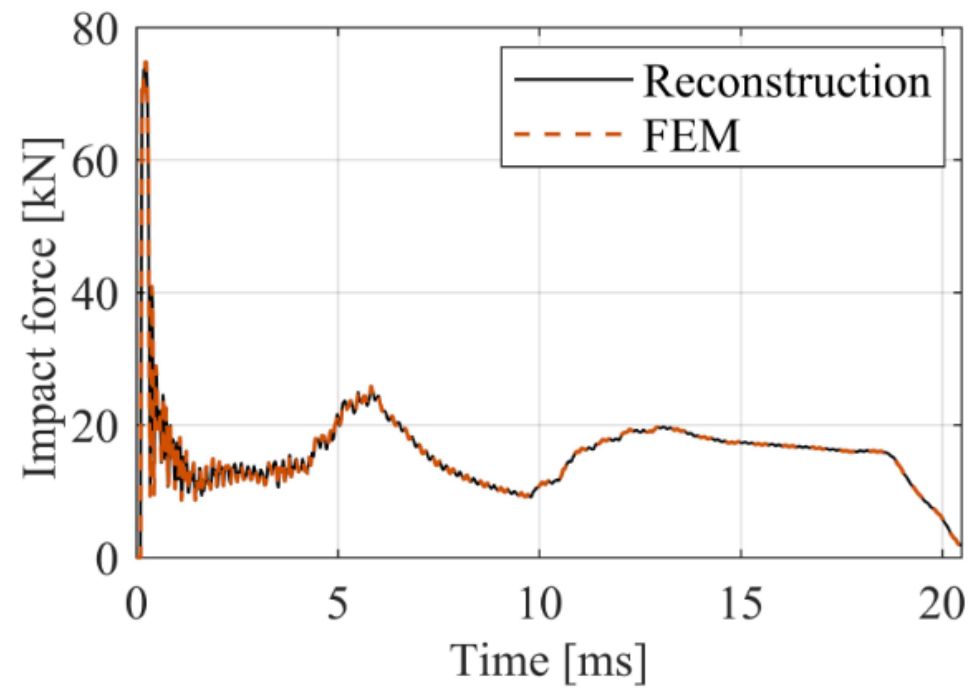

(a) The whole impact time

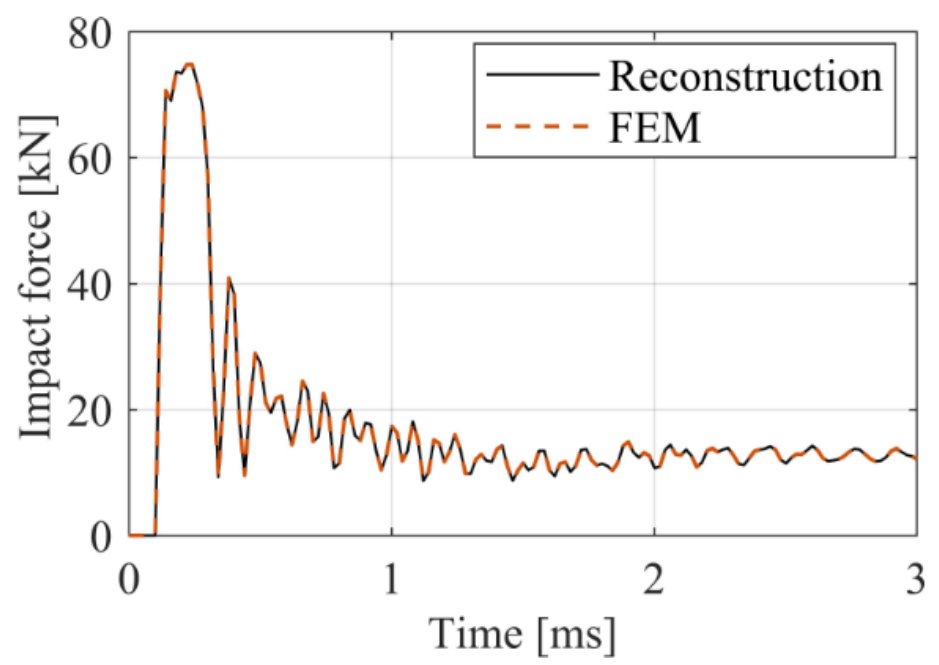

(b) Magnification for the first $3 \mathrm{~ms}$

Figure 5. Reconstructed impact force for square tube: $100 \mathrm{~mm}$ long $\times 1.0 \mathrm{~mm}$ thick $\times 46$ mm square cross-section, and $V=6 \mathrm{~m} / \mathrm{s}$ using four responses

On the other hand, the conventional deconvolution described above is also applied in order to reconstruct the impact force and compare with the wavelet technique. Note that both deconvolution techniques are deployed in terms of using four response locations as pointed out in Figure 2(b). This comparison is exhibited in Figure 6. As seen in Figure 6(a), although both deconvolution techniques are good enough when estimating well the impact force, the conventional deconvolution still reveals some noise expansion in reconstructed result. This disadvantage can be clearly observed in Figure 6(b) where the comparison of 
impact force between two techniques is zoomed in from 11 to 19 ms of impact time. Accordingly, the wavelet technique shows a perfect match with FEM result, whereas there is perturbation with the conventional deconvolution although no measurement error is assumed in this simulation.

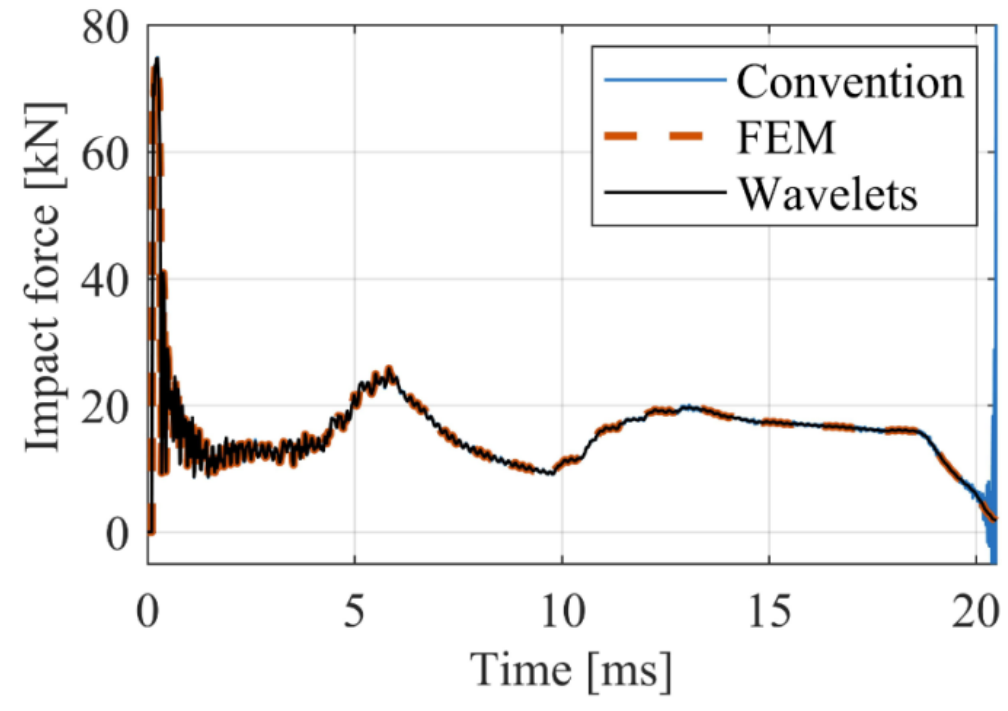

(a) The whole impact time

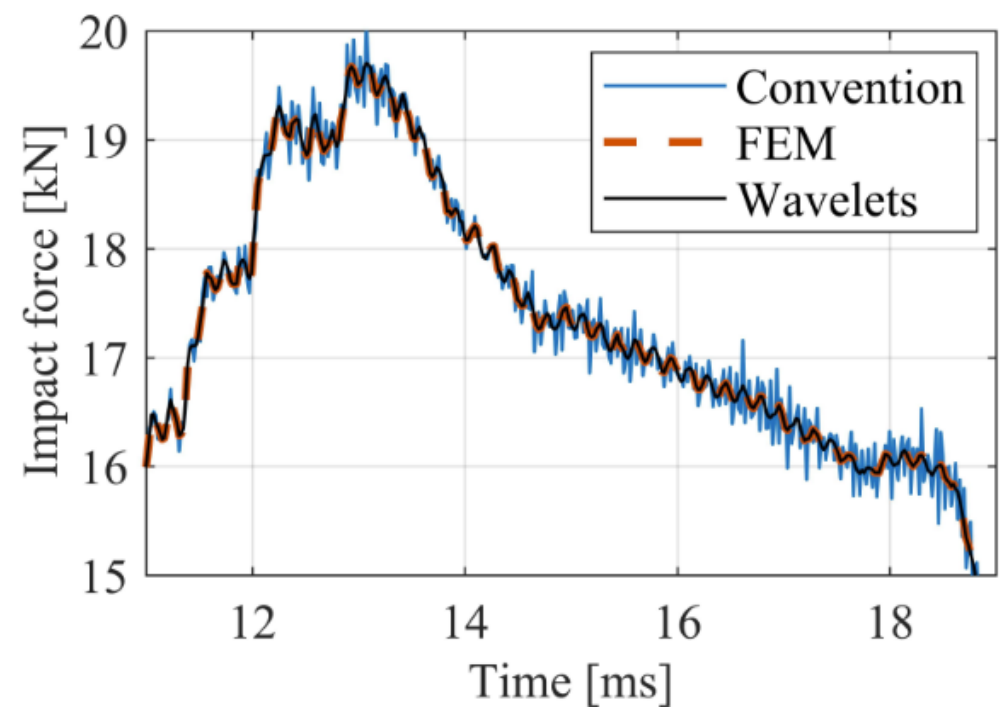

(b) Magnification from 11 to 19 ms of impact time

Figure 6. Comparison of reconstructed impact force for square tube: $100 \mathrm{~mm}$ long $\times 1.0$ $\mathrm{mm}$ thick $\times 46 \mathrm{~mm}$ square cross-section, and $V=6 \mathrm{~m} / \mathrm{s}$ between the wavelet and conventional deconvolution technique

In the examples shown in Figures 4 and 5, however, the reconstructed impact force is the same one used in determining the impulse response function. It should be examined whether the impact force can be reconstructed successfully even when the impulse response function is determined under different conditions such as changing in length or thickness of the square tube, and varying impact velocity. Figure 7 shows the results of reconstruction for the cases of different size tubes $(180 \mathrm{~mm}$ long $\times 0.8$ or $1.0 \mathrm{~mm}$ thick $\times 46 \mathrm{~mm}$ square cross-section) using the impulse response function determined for the original size tube (100 
$\mathrm{mm}$ long $\times 1.0 \mathrm{~mm}$ thick $\times 46 \mathrm{~mm}$ square cross-section). It can be seen that the impact forces are reconstructed very well in both cases. In addition, the impact forces for different impact velocities $(V=1,2,3,4$, and $5 \mathrm{~m} / \mathrm{s})$ are reconstructed almost perfectly using the impulse response function determined at $V=6 \mathrm{~m} / \mathrm{s}$, which are shown in Figure 8. The reconstructed impact forces in the time range of $3 \mathrm{~ms}$ as figured out in Figures 4(b), 5(b), and insets in Figure 7 show an excellent match with FEM results at the peak portion of impact force. Further, because the folding duration of a tube under impact load is an important factor in order to assess its energy absorption capability, it should be noted that the different impact durations are also reconstructed accurately in any cases with wavelet deconvolution technique. In the cases of different impact velocity, a few error expansions have occurred at the very end of the time range computed, which is due to the traveling time of elastic waves from impact site to the strain measurement location but is negligible in practice. As a result, it can be concluded that the wavelet deconvolution technique works very well if multiple responses are employed for reconstruction.

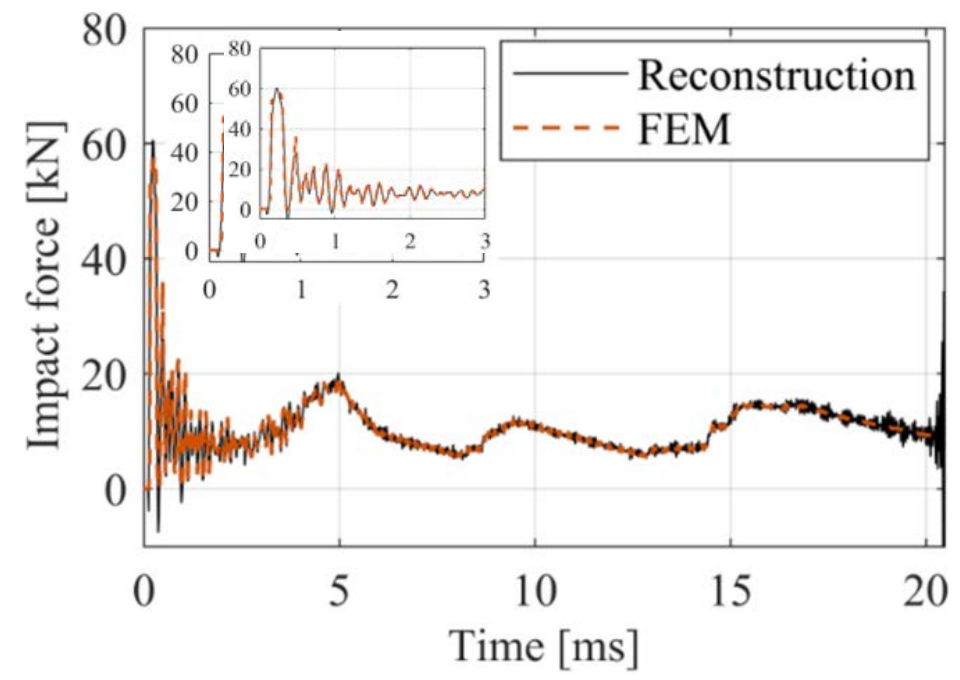

(a) $180 \mathrm{~mm}$ long $\times 0.8 \mathrm{~mm}$ thick $\times 46 \mathrm{~mm}$ square cross-section

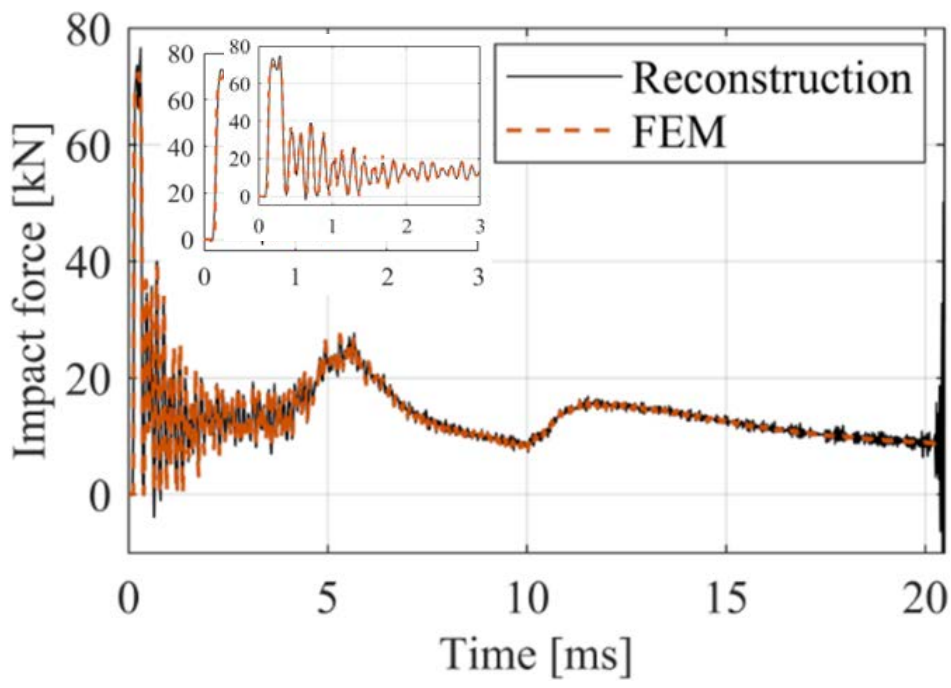

(b) $180 \mathrm{~mm}$ long $\times 1.0 \mathrm{~mm}$ thick $\times 46 \mathrm{~mm}$ square cross-section

Figure 7. Reconstructed impact forces for the square tube when $V=6 \mathrm{~m} / \mathrm{s}$ 


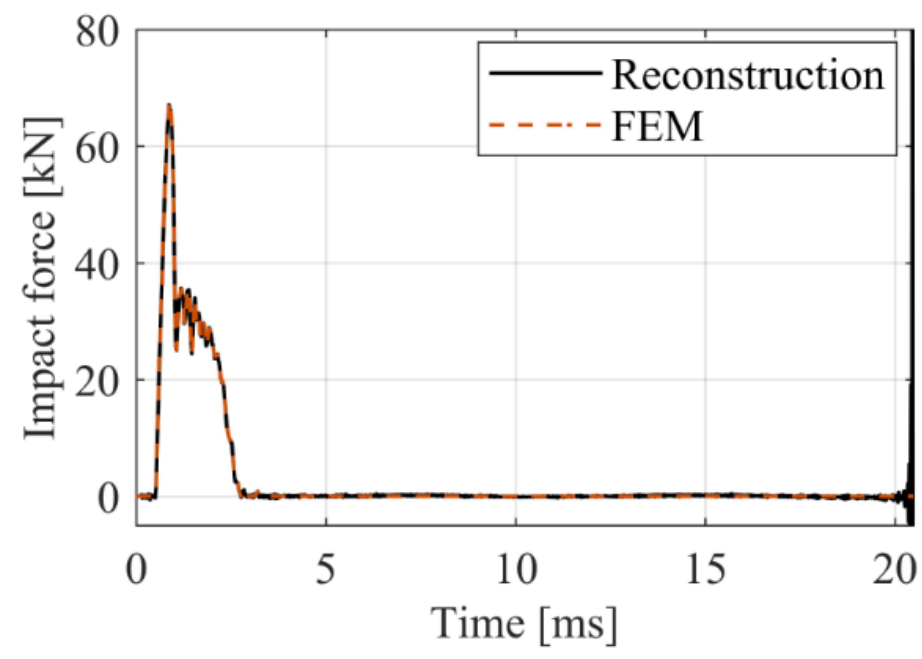

(a) At $V=1 \mathrm{~m} / \mathrm{s}$

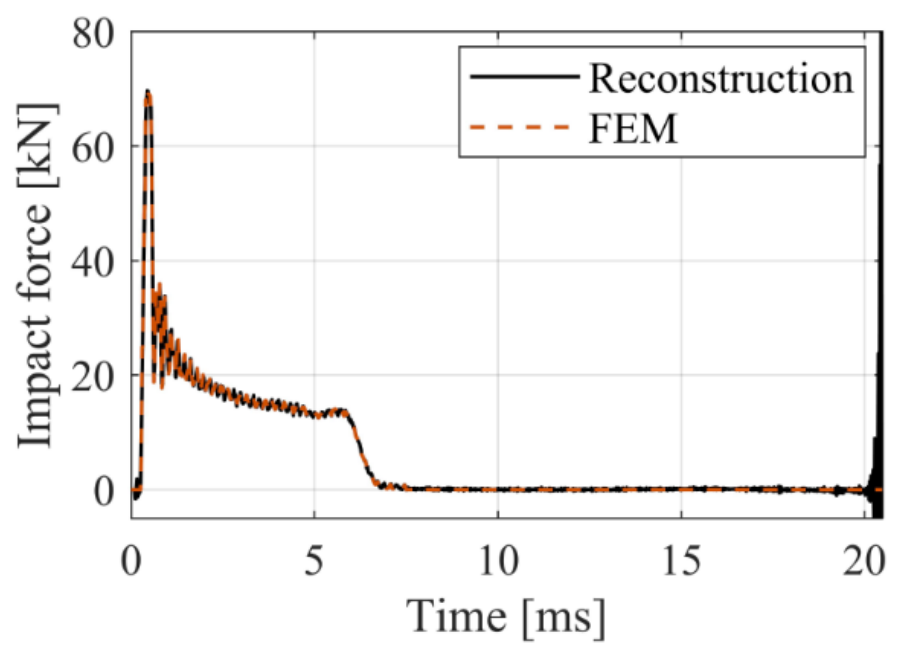

(b) At $V=2 \mathrm{~m} / \mathrm{s}$

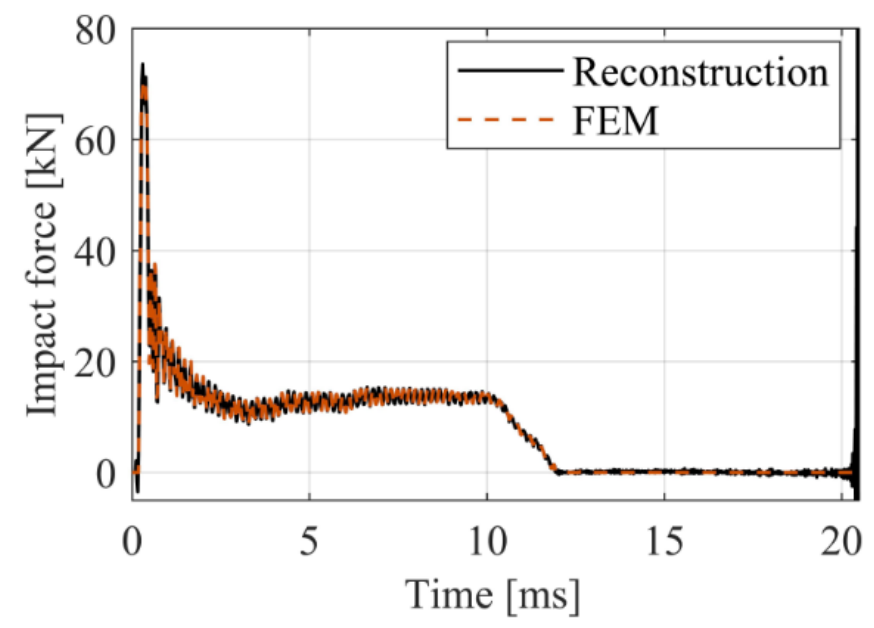

(c) At $V=3 \mathrm{~m} / \mathrm{s}$

Figure 8. Reconstructed impact forces for square tube: $100 \mathrm{~mm}$ long $\times 1.0 \mathrm{~mm}$ thick $\times 46$ mm square cross-section with different impact velocity (to be continued) 


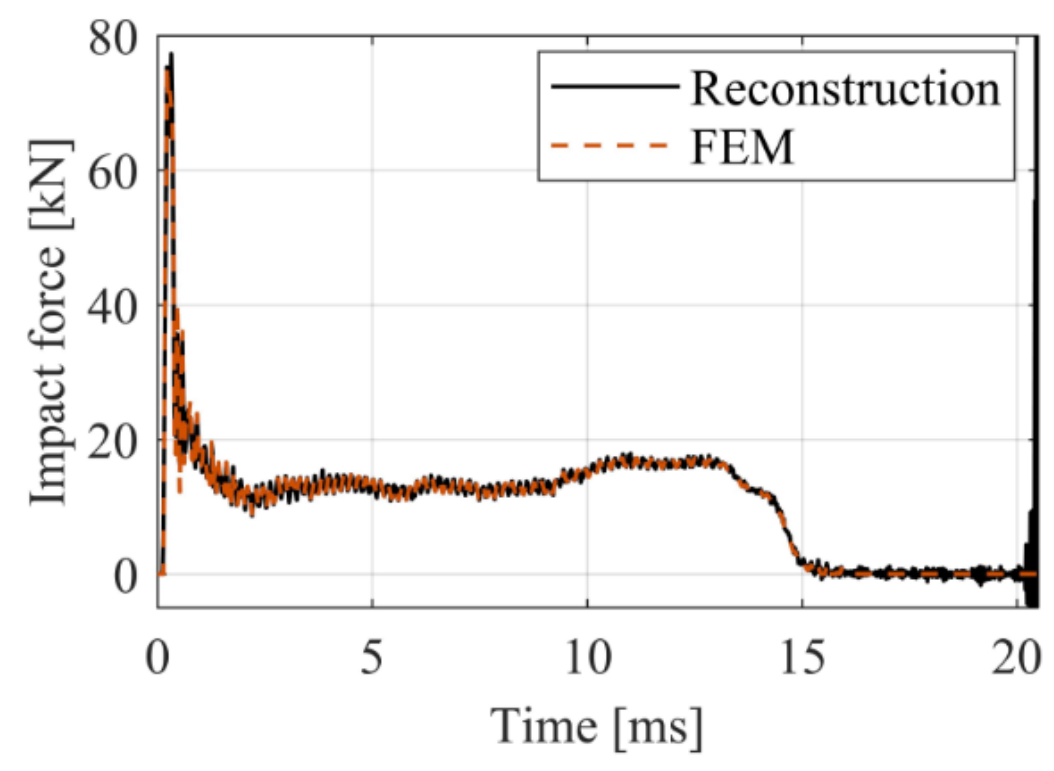

(d) At $V=4 \mathrm{~m} / \mathrm{s}$

Figure 8. Continued

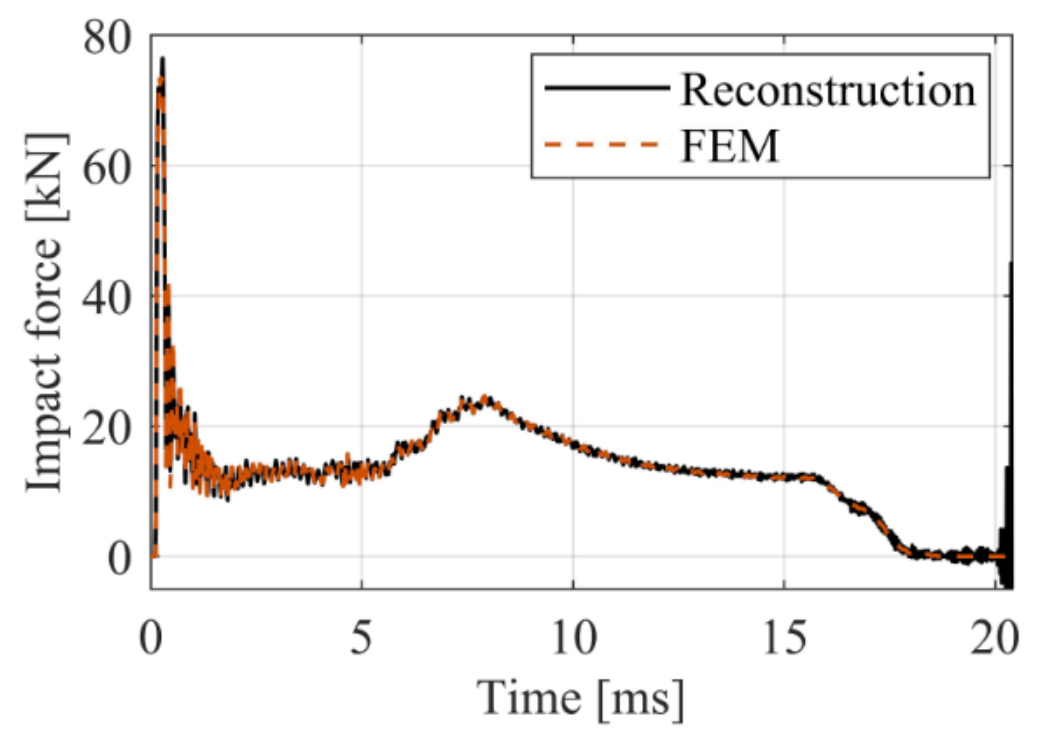

(e) $V=5 \mathrm{~m} / \mathrm{s}$

Figure 8. Continued

\section{Conclusions}

In this research, the impact force reconstruction using the wavelet deconvolution technique has been proposed. The comparisons between FEA and reconstruction results have demonstrated the effectiveness of the wavelet deconvolution technique. The proposed technique reconstructed the impact force successfully and accurately despite changing the impact velocity or the length and thickness of the thin-walled column. For further development, first, the reconstruction capability of the present technique needs to be examined in the case when some measurement errors are included in the impact response. Second, the applicability of regularization techniques should be considered to reduce the influences of errors on the reconstruction of impact force. 


\section{References}

[1] H. Inoue, J.J. Harrigan, and S.R. Reid, "Review of inverse analysis for indirect measurement of impact force," Applied Mechanics Reviews, Vol. 54, pp. 503-524, 2001.

[2] J. Sanchez, and H. Benaroya, "Review of force reconstruction techniques,” Journal of Sound and Vibration, Vol. 333, No. 14, pp. 2999-3018, 2014.

[3] E. Wu, T.D. Tsai, and C.S. Yen, "Two methods for determining impact-force history on elastic plates,” Experimental Mechanics, Vol. 35, No. 1, pp. 11-18, 1995.

[4] S.Y. Khoo, Z. Ismail, K.K. Kong, Z.C. Ong, S. Noroozi, W.T. Chong, and A.G.G. Rahman, "Impact force identification with pseudo-inverse method on a lightweight structure for under-determined, even-determined and over-determined cases," International Journal of Impact Engineering, Vol. 63, pp. 52-62, 2014.

[5] E.F. Gunawan, "Impact force reconstruction using the regularized Wiener filter method," Inverse Problems in Science and Engineering, Vol. 24, No. 7, pp. 1107-1132, 2015.

[6] B.J. Qiao, X.W. Zhang, X.J. Luo, and X.F. Chen, “A force identification method using cubic B-spline scaling functions,” Journal of Sound and Vibration, Vol. 337, pp. 28-44, 2015.

[7] J.F. Doyle, “A wavelet deconvolution method for impact force identification," Experimental Mechanics, Vol. 37, No. 4, pp. 403-408, 1997.

[8] J.F. Doyle, "Reconstructing dynamic events from time-limited spatially distributed data," International Journal for Numerical Methods in Engineering, Vol. 53, pp. 2721-2734, 2002.

[9] I. Daubechies, Ten Lectures on Wavelets, SIAM, Philadelphia, United States of America, 1992.

[10] H. Inoue, S. Yokoyama, K. Kishimoto, and T. Shibuya, "Inverse analysis of impact force using wavelet transform," In M. Tanaka (Chair), International Symposium on Inverse Problems in Engineering Mechanics, February, 2001.

[11] L.A. Lifschitz, and C.E. D’Attellis, "Input force reconstruction using wavelets with application to a pulsed plasma thruster," Mathematical and Computer Modeling, Vol. 41, pp. 361-369, 2005.

[12] Z. Li, Z.P. Feng, and F.L. Chu, "A load identification method based on wavelet multiresolution analysis,” Journal of Sound and Vibration, Vol. 333, No. 2, pp. 381-391, 2014.

[13] F.E. Gunawan, H. Homma, and Y. Kanto, “Two-step B-splines regularization method for solving an ill-posed problem of impact-force reconstruction,” Journal of Sound and Vibration, Vol. 297, No. 1, pp. 200-214, 2006.

[14] A. Jusuf, T. Dirgantara, L. Gunawan, and I.S. Putra, "Crashworthiness analysis of multi-cell prismatic structures,” International Journal of Impact Engineering, Vol. 78, pp. 34-50, 2015.

[15] L. Gunawan, T. Dirgantara, I.S. Putra, and V.C. Thanh, "Development of load cell for low velocity axial impact tests," In: Proceeding of Regional Conference on Mechanical and Aerospace Technology, Bali, Indonesia, No. 320, pp. 1-7, 2010. 